\title{
Inoculação de proteína isolada de soja em ovos embrionados oriundos de matrizes semipesadas com diferentes idades
}

\author{
[Isolated soy protein inoculation in embryonated eggs of semi heavy from \\ matrices with different ages] \\ J.L. Damasceno ${ }^{1}$, F.G.G. Cruz $^{2 *}$, R.D. Melo ${ }^{2}$, J.C. Feijó ${ }^{3}$, J.P.F. Rufino ${ }^{4}$, \\ F.M. Valentim ${ }^{5}$, J.P.C. Oliveira ${ }^{5}$

\footnotetext{
${ }^{1}$ Aluna de pós-graduação - Universidade Estadual do Oeste do Paraná - Marechal Cândido Rondon, PR ${ }^{2}$ Faculdade de Ciências Agrárias - Universidade Federal do Amazonas - Manaus, AM

${ }^{3}$ Aluno de graduação - Faculdade de Ciências Agrárias - Universidade Federal do Amazonas - Manaus, AM

${ }^{5}$ Zootecnista autônomo - Manaus, AM
} \\ ${ }^{4}$ Aluno de pós-graduação - Faculdade de Ciências Agrárias - Universidade Federal do Amazonas - Manaus, AM
}

\begin{abstract}
RESUMO
Este estudo objetivou avaliar os efeitos da inoculação de proteína isolada de soja (P.I.S.) em ovos embrionados de matrizes semipesadas com diferentes idades. Foram utilizados 320 ovos embrionados de matrizes semipesadas da linhagem Rhode Island Red com 35 e 70 semanas de idade. Ao $17^{\circ}$ dia de incubação, os ovos foram inoculados com $0,5 \mathrm{~mL}$ de solução diretamente na cavidade alantoide. $\mathrm{O}$ delineamento experimental utilizado foi o inteiramente ao acaso, em esquema fatorial $(2 \times 4)$, constituído por dois fatores: idades das matrizes (matrizes jovens e matrizes velhas) e soluções inoculadas (ovo íntegro; $0,5 \%$ de solução salina; $1 \%$ de P.I.S. $+0,5 \%$ de solução salina; e $2 \%$ de P.I.S. $+0,5 \%$ de solução salina), totalizando oito tratamentos contendo 40 ovos cada. Os dados coletados foram analisados pelo teste de Tukey a $5 \%$ de significância. A inoculação de proteína isolada de soja apresentou efeito positivo sobre a relação pinto-ovo, porém sem proporcionar melhora nos rendimentos de incubação, no desenvolvimento dos órgãos e no desempenho de pintos na fase pré-inicial. A idade da matriz influenciou diretamente as variáveis avaliadas, em que ovos oriundos de aves velhas apresentaram maior peso, maior mortalidade embrionária na fase tardia, pintos mais pesados e pior conversão alimentar.
\end{abstract}

Palavras-chave: desempenho de pintos, eclodibilidade, mortalidade embrionária, nutrição in ovo

\begin{abstract}
This study aimed to evaluate the influence of inoculation of isolated soy protein (P.I.S.) in embryonated eggs of semi-heavy with different ages. 320 embryonated eggs were used from semi-heavy breeders of Rhode Island Red lineage with 35 and 70 weeks of age. In the 17th day of incubation, the eggs were inoculated with $0.5 \mathrm{ml}$ of solution in the allantoic cavity. The experimental method was completely randomized in factorial scheme $(2 \times 4)$, consisting of two factors: matrices ages (young and old matrices) and inoculation solutions (non-inoculated eggs; $0.5 \%$ of saline solution; $1 \%$ of P.I.S. $+0.5 \%$ of saline solution; and $2 \%$ of P.I.S. $+0.5 \%$ of saline solution, totalling eight treatments containing 40 eggs each. The inoculation of isolated soy protein showed positive effect on the chick-egg relation, albeit without improving incubation yields, organ development and chicks's performance in the pre-initial phase. The age of the breeder directly influenced the variables evaluated, with eggs from older breeder showing better weight, higher embryonic mortality in late phase, heavier chicks and worse feed conversion.
\end{abstract}

Keywords: chick performance, embryo mortality, hatchability, in ovo nutrition

Recebido em 5 de novembro de 2016

Aceito em 6 de janeiro de 2017

*Autor para correspondência (corresponding author)

E-mail: frankgcruz@gmail.com 


\section{INTRODUÇÃO}

A incubação é uma das mais importantes etapas na indústria avícola moderna, pois tem como objetivo produzir pintos saudáveis, logo, a partir destes, são obtidos os principais produtos oriundos da supracitada indústria (Freitas et al., 2010). Dessa forma, nesse segmento específico da avicultura, diversos aspectos relacionados à matriz são evidenciados como fator de influência à qualidade interna e externa do ovo, ao peso do ovo e à qualidade do pinto de um dia.

Esses parâmetros de avaliação, associados a fatores como a idade da matriz, podem explicar, por exemplo, por que pintos provenientes de matrizes jovens têm mortalidade aumentada e desempenho reduzido (Vieira e Moran Jr., 1998), enquanto $\mathrm{o}$ aumento da idade da matriz determina maior perda de peso em ovos na incubação, com elevação da taxa de mortalidade embrionária e consequente queda da eclodibilidade (McDaniel et al., 1979). Nesse aspecto, a nutrição in ovo surge como uma ferramenta que pode auxiliar e trazer benefícios ao processo de incubação, podendo ser um método alternativo às empresas avícolas para melhorarem seus resultados, pois o aumento de apenas uma unidade na eclodibilidade já representa grande valor econômico para as empresas (Ipek et al., 2004).

Essa tecnologia apresenta ainda a possibilidade de desenvolvimento precoce das aves, que, caso contrário, só ocorreria após a eclosão. Este consiste ainda no fornecimento de nutrientes para o pinto durante o seu desenvolvimento embrionário, com a finalidade de aumentar o estado nutricional do embrião, além de permitir a introdução de nutrientes específicos em contato com as células do intestino, melhorando, assim, a capacidade de digerir alimentos pelo neonato, pois, após a eclosão, as aves apresentam funções digestivas limitadas, o que diminui, inicialmente, a disponibilidade de nutrientes para seu crescimento $\mathrm{e}$ as torna susceptíveis aos patógenos (Campos et al., 2010).

Além disso, apesar de pintinhos, ao nascimento, apresentarem uma reserva energética no saco vitelínico, essa reserva não é suficiente para suprir a necessidade energética de manutenção dos animais nem mesmo durante o primeiro dia de vida (Santos, 2007). Isso porque a concentração de carboidratos é extremamente baixa, com menos de $1 \%$ do total, e apenas $0,3 \%$ desse total é glicose livre.

Nesse contexto, a inoculação de substâncias, como a proteína isolada de soja in ovo, pode ser benéfica ao embrião por proporcionar uma maior demanda de aminoácidos, especialmente os de cadeia ramificada (valina, leucina e isoleucina), que, apesar de não serem considerados a principal fonte de energia para a contração muscular, atuam como uma importante fonte de energia para o músculo esquelético durante períodos de estresse metabólico (Alves, 2002), fornecendo ao embrião um aporte nutricional para auxiliá-lo na sua fase final de desenvolvimento. Logo, este estudo objetivou analisar a inoculação de proteína isolada de soja em ovos embrionados oriundos de matrizes semipesadas com diferentes idades sobre os rendimentos de incubação, os percentuais de mortalidade embrionária, o desenvolvimento do trato gastrointestinal e o desempenho dos pintos.

\section{MATERIAL E MÉTODOS}

Este estudo foi conduzido no Laboratório de Tecnologia Avícola do Setor de Avicultura da Faculdade de Ciências Agrárias da Universidade Federal do Amazonas. O experimento e todos os procedimentos utilizados foram aprovados pelo Comitê de Ética no Uso de Animais (Ceua) da Universidade Federal do Amazonas, sob o protocolo $n^{\circ} 033 / 2015$.

Foram incubados 200 ovos de matrizes da linhagem Rhode Island Red, com 35 semanas de idade, e 200 ovos de matrizes da mesma linhagem, com 70 semanas de idade. Esses ovos foram pesados, identificados e incubados em incubadora modelo PETERSIME 168, com temperatura de $37,5^{\circ} \mathrm{C}$ e $66 \%$ de umidade relativa do ar. sendo efetuada a viragem dos ovos em intervalos de uma hora.

Ao $17^{\circ}$ dia de incubação, utilizando-se um ovoscópio, selecionaram-se 160 ovos embrionados oriundos de cada uma das idades predeterminadas, os quais foram distribuídos em delineamento experimental inteiramente ao acaso (DIC), em esquema fatorial $2 \times 4$, constituído de dois fatores: idades das matrizes (ovos de matrizes jovens e ovos de matrizes velhas) e concentrações de inoculação de proteína isolada 
de soja (ovo íntegro; $0,5 \%$ de solução salina; $1 \%$ de proteína isolada de soja $+0,5 \%$ de solução salina; e $2 \%$ de proteína isolada de soja $+0,5 \%$ de solução salina), totalizando oito tratamentos contendo 40 ovos cada, sendo cada ovo considerado uma repetição.

As soluções testadas foram desenvolvidas com base em solução salina $0,5 \%$, produzida com água deionizada estéril e cloreto de sódio $(\mathrm{NaCl})$, na qual foi incluído cada componente nutricional na dosagem necessária para se obter, no final a concentração desejada, conforme descrito por Campos et al. (2011).

Para a inoculação, os ovos foram higienizados com álcool iodado ( $2 \%$ ) na região da câmara de ar e, então, perfurados mediante a utilização de agulhas estéreis de $0,80 \times 25 \mathrm{~mm}$ ( $21 \mathrm{~g} \times 1$ ”), evitando-se perfurar a membrana interna da casca do ovo. Em cada ovo, inoculou-se $0,5 \mathrm{~mL}$ de solução contendo os nutrientes avaliados, diretamente na cavidade alantoide, utilizando-se seringas de $1 \mathrm{~mL}$ e agulhas de 13 x $0,45 \mathrm{~mm}$ (26G x $1 / 2$ "), estéreis, para cada ovo. O orifício perfurado na casca foi vedado com parafina fundida, e os ovos foram embalados individualmente em sacos de organza e transferidos diretamente para nascedouro modelo PETERSIME 168, onde a máquina manteve a temperatura de $36,5^{\circ} \mathrm{C}$ e $76 \%$ de umidade relativa do ar.

Os pintos, por ocasião do nascimento $(504 \pm 2$ horas de incubação), e suas respectivas cascas foram pesados para determinação da perda de peso ao final da incubação. O peso dos pintos foi utilizado para determinação de sua relação com o peso do ovo (Pedroso et al., 2006).

A taxa de eclodibilidade de cada tratamento foi determinada por meio da relação entre o número de ovos eclodidos pelo total de ovos férteis incubados. Ao final do período de incubação, os ovos não eclodidos foram abertos para determinação do período de mortalidade embrionária. A mortalidade embrionária foi classificada em intermediária (17 a 18 dias), tardia (19 a 21 dias, sem bicagem da casca), pósbicagem da casca (19 a 21 dias, com bicagem da casca) e pós-nascimento (pintos descartados).

Em seguida, foram selecionados e abatidos cinco pintos aptos de cada tratamento, os quais foram eutanasiados por deslocamento cervical para realização da biometria dos órgãos do trato gastrointestinal, sendo analisadas as seguintes variáveis: peso do saco vitelino, peso do fígado, peso do esôfago + papo, peso do pró-ventrículo + moela, peso dos intestinos delgado e grosso, comprimento dos intestinos delgado e grosso e comprimento total do sistema digestivo.

Para avaliação do desempenho dos pintos, foram selecionados 25 pintos aptos de cada tratamento, sendo esses vacinados (Marek, Bouba Aviária e Gumboro) e alojados em baterias metálicas aquecidas conforme a distribuição dos tratamentos. Todas as aves receberam a mesma dieta, formulada segundo recomendações de Rostagno et al. (2011), com ração e água ad libitum. As aves e as rações foram pesadas diariamente durante 10 dias a fim de se mensurarem as seguintes vaiáveis: consumo de ração, ganho de peso, conversão alimentar, peso final e viabilidade.

Os dados coletados foram submetidos à análise de variância e as médias estimadas foram avaliadas pelo teste Tukey a 5\% de significância, por meio do procedimento GLM do programa computacional Statistical Analysis System (2008).

\section{RESULTADOS E DISCUSSÃO}

Os resultados médios obtidos para eclodibilidade e mortalidade embrionária estão dispostos na Tab. 1. A inoculação de proteína isolada de soja e a idade da matriz não afetaram significativamente a eclodibilidade $(\mathrm{P}>0,05)$ dos ovos. Todavia, na mortalidade embrionária, ovos inoculados apresentaram diferenças significativas $(\mathrm{P}<0,05)$ no percentual de embriões mortos na fase pós-bicagem, bem como ovos oriundos de matrizes com diferentes idades na fase tardia.

Constatou-se que, a partir da inoculação de proteína isolada de soja consorciada com solução placebo, houve redução gradativa das taxas de eclodibilidade e consequentemente, aumento da mortalidade embrionária, principalmente intermediária (logo após a inoculação). Esses resultados corroboram os observados por Pedroso et al. (2006), que verificaram aumento na mortalidade embrionária quando realizada a inoculação de solução de 
glicose no líquido amniótico aos 16 dias de incubação. Segundo Ohta e Kidd (2001) e Jochemsen e Jeurissen (2002), diversos fatores são responsáveis por ocasionar elevadas taxas de mortalidade embrionária em ovos após a inoculação de substâncias, nos quais se destacam a idade, o peso e o tempo de armazenamento do ovo, o local de inoculação, a idade da matriz, dentre outros. Pedroso et al. (2006) descrevem ainda a osmolaridade e a própria composição bioquímica das substâncias como fatores diretamente responsáveis por ocasionar mortalidade embrionária, principalmente em períodos logo após a inoculação.

Tabela 1. Eclodibilidade e mortalidade embrionária em ovos oriundos de matrizes com diferentes idades e inoculados ou não com proteína isolada de soja

\begin{tabular}{|c|c|c|c|c|c|}
\hline \multirow[b]{2}{*}{ Tratamentos } & \multirow{2}{*}{$\begin{array}{l}\text { Eclodibilidade } \\
(\%)\end{array}$} & \multicolumn{4}{|c|}{ Mortalidade embrionária (\%) } \\
\hline & & Intermediária & Tardia & Pós-bicagem & Pós-nascimento \\
\hline \multicolumn{6}{|l|}{ Soluções inoculadas } \\
\hline Ovo íntegro & 90,00 & 2,50 & 5,00 & $0,00 \mathrm{a}$ & 2,50 \\
\hline Placebo & 88,75 & 2,50 & 2,50 & $3,75 b$ & 2,50 \\
\hline 1\% P.I.S. & 87,50 & 1,25 & 3,75 & $2,5 \mathrm{ab}$ & 5,00 \\
\hline $2 \%$ P.I.S. & 88,75 & 1,25 & 3,75 & $2,5 \mathrm{ab}$ & 3,75 \\
\hline \multicolumn{6}{|l|}{ Idade da matriz } \\
\hline Aves jovens & 89,38 & 2,5 & $1,87 \mathrm{a}$ & 1,25 & 5,00 \\
\hline Aves velhas & 88,13 & 1,25 & $5,62 b$ & 3,12 & 1,88 \\
\hline \multicolumn{6}{|l|}{ Efeito } \\
\hline Soluções inoculadas & $0,35^{\mathrm{ns}}$ & $0,83^{\mathrm{ns}}$ & $0,51^{\mathrm{ns}}$ & $0,04 *$ & $0,52^{\mathrm{ns}}$ \\
\hline Idade da matriz & $0,20^{\mathrm{ns}}$ & $0,37^{\mathrm{ns}}$ & $0,01 *$ & $0,24^{\mathrm{ns}}$ & $0,07^{\mathrm{ns}}$ \\
\hline Interação & $0,34^{\mathrm{ns}}$ & $0,01 *$ & $0,23^{\mathrm{ns}}$ & $0,22^{\mathrm{ns}}$ & $0,20^{\mathrm{ns}}$ \\
\hline $\mathrm{CV}(\%)$ & 12,75 & 16,33 & 27,22 & 12,12 & 4,92 \\
\hline
\end{tabular}

CV - Coeficiente de variação. * Médias seguidas por letras minúsculas na coluna diferem entre si pelo teste Tukey a $5 \%(\mathrm{P}<0,05)$; ns - não significativo.

A interação entre os fatores (Tab. 2) também apresentou diferenças significativas $(\mathrm{P}<0,05)$ nos percentuais de mortalidade intermediária. Quando observados esses resultados, verifica-se a existência de uma relação entre embriões oriundos de ovos de matrizes mais velhas e inoculados com os maiores percentuais de proteína isolada de soja, em que estes apresentaram maior mortalidade logo após a inoculação, o que evidencia que a idade da matriz pode influenciar negativamente a absorção de nutrientes exógenos e, consequentemente, ocasionar a morte do embrião. Esses resultados são semelhantes aos observados por Meijerhof et al. (1994), que, ao utilizarem ovos de matrizes com diferentes idades, verificaram que a eclodibilidade dos ovos diminuiu com o aumento do período de armazenamento e que os ovos das matrizes velhas, quando comparados com matrizes jovens, apresentaram maior taxa de mortalidade durante o processo de incubação, resultando em menor percentual de pintos eclodidos.

Tabela 2. Interação entre os fatores sobre a mortalidade intermediária de embriões oriundos de ovos de matrizes com diferentes idades, inoculados ou não com proteína isolada de soja

\begin{tabular}{|c|c|c|}
\hline \multirow{3}{*}{$\begin{array}{l}\text { Soluções } \\
\text { inoculadas }\end{array}$} & \multicolumn{2}{|c|}{ Idade da matriz } \\
\hline & Aves jovens & Aves velhas \\
\hline & \multicolumn{2}{|c|}{ Mortalidade intermediária (\%) } \\
\hline \multicolumn{3}{|l|}{ Ovo } \\
\hline íntegro & $2,50 \mathrm{Ba}$ & $0,00 \mathrm{Ab}$ \\
\hline Placebo & $2,50 \mathrm{Ba}$ & $0,00 \mathrm{Ab}$ \\
\hline $1 \%$ P.I.S. & $0,00 \mathrm{Aa}$ & $1,25 \mathrm{Bb}$ \\
\hline $2 \%$ P.I.S. & $0,00 \mathrm{Aa}$ & $1,25 \mathrm{Bb}$ \\
\hline
\end{tabular}

$\mathrm{CV}(\%)$

CV - Coeficiente de variação. * Médias seguidas por letras diferentes, maiúsculas na coluna (efeito entre idades) e minúsculas na linha (efeito entre soluções) diferem entre si pelo teste Tukey a $5 \%$. 
Os resultados médios obtidos para as variáveis da relação ovo-pinto estão dispostos na Tab. 3. A inoculação da proteína isolada de soja influenciou significativamente $(\mathrm{P}<0,05)$ a relação peso do pinto sobre o peso do ovo. Constatou-se que a inoculação de nutrientes exógenos beneficiou a relação entre o peso do pinto e o peso do ovo nos diferentes tratamentos aplicados, sendo os melhores valores atribuídos aos ovos que foram inoculados somente com solução salina. Esses resultados corroboram os observados por Uni e Ferket (2004) e Leitão et al. (2010), que, em inoculação de carboidratos in ovo, não observaram efeito significativo na relação ovo-pinto, todavia verificaram incremento no peso de pintos oriundos de ovos inoculados.
Entre idade da matriz, observou-se efeito significativo $(\mathrm{P}<0,05)$ sobre o peso do ovo, a perda de peso do ovo e o peso do pinto, em que os ovos oriundos de matrizes jovens apresentaram-se menores, com maior perda de peso verificada no momento da transferência e pintos com menor peso ao nascer. De acordo com McLoughlin e Gous (2000), esses resultados encontram-se diretamente relacionados a uma quantidade menor de poros na casca e na membrana, cutícula mais espessa, albúmen mais viscoso e menor fonte de nutrientes que os ovos menores, principalmente quando oriundos de matrizes jovens, o que contribui para a eclosão de pintos menores. Em contrapartida, com o aumento da idade das aves, a casca, a cutícula e as membranas tornam-se mais finas, o que melhora a concentração de nutrientes na gema, e, consequentemente, nascem pintos maiores.

Tabela 3. Peso do ovo (PO), perda de peso do ovo ao final da incubação (PPO), peso dos pintos ao nascer (PP) e relação entre o peso do pinto e o peso do ovo (PP/PO) em ovos oriundos de matrizes com diferentes idades e inoculados ou não com proteína isolada de soja

\begin{tabular}{|c|c|c|c|c|}
\hline \multirow{2}{*}{ Tratamentos } & \multicolumn{4}{|c|}{ Variáveis } \\
\hline & PO $(g)$ & PPO (\%) & $\mathrm{PP}(\mathrm{g})$ & $\mathrm{PP} / \mathrm{PO}$ \\
\hline \multicolumn{5}{|l|}{ Soluções inoculadas } \\
\hline Ovo íntegro & 52,83 & 9,57 & 33,58 & $63,77^{b}$ \\
\hline Placebo & 51,98 & 9,68 & 34,52 & $66,63^{\mathrm{a}}$ \\
\hline $1 \%$ P.I.S. & 52,10 & 9,65 & 34,12 & $65,56^{\mathrm{ab}}$ \\
\hline 2\% P.I.S. & 51,91 & 9,80 & 34,25 & $66,09^{\mathrm{ab}}$ \\
\hline \multicolumn{5}{|l|}{ Idade da matriz } \\
\hline Aves jovens & $48,58^{\mathrm{b}}$ & $9,80^{\mathrm{a}}$ & $32,00^{b}$ & 65,98 \\
\hline Aves velhas & $55,83^{\mathrm{a}}$ & $9,54^{\mathrm{b}}$ & $36,24^{\mathrm{a}}$ & 65,04 \\
\hline \multicolumn{5}{|l|}{ Efeito } \\
\hline Soluções inoculadas & $0,40^{\mathrm{ns}}$ & $0,64^{\mathrm{ns}}$ & $0,33^{\text {ns }}$ & $0,02 *$ \\
\hline Idade da matriz & $0,01^{*}$ & $0,04 *$ & $0,01^{*}$ & $0,16^{\mathrm{ns}}$ \\
\hline Interação & $0,23^{\text {ns }}$ & $0,05^{\mathrm{ns}}$ & $0,43^{\text {ns }}$ & $0,23^{\text {ns }}$ \\
\hline $\mathrm{CV}(\%)$ & 2,59 & 4,14 & 3,37 & 3,20 \\
\hline
\end{tabular}

Não houve resultados significativos $(\mathrm{P}>0,05)$ da inoculação dos nutrientes sobre a biometria dos órgãos (Tab. 4). Todavia, entre ovos oriundos de matrizes com diferentes idades, observaram-se resultados significativos $(\mathrm{P}<0,05)$, em que os pintos eclodidos de ovos de matrizes mais velhas apresentaram melhores resultados de peso do saco vitelino, de peso e comprimento dos intestinos e no comprimento total do sistema digestivo.

Segundo Vick et al. (1993), ovos provenientes de matrizes mais velhas normalmente apresentam maior peso da gema e, por consequência, maior saco vitelino e maior quantidade de nutrientes para o pinto ao nascimento. Luquetti et al. (2004) 
observaram que reprodutoras mais velhas produziram pintos com maior peso corporal ao nascimento e maior desenvolvimento em peso absoluto de órgãos. O mesmo fato ocorreu com o desenvolvimento do intestino delgado, observado por Maiorka et al. (2000), os quais, ao avaliarem o crescimento desse órgão, em pintos provenientes de matrizes com diferentes idades, observaram que os provenientes de matrizes velhas apresentaram maior comprimento e maior peso relativo do intestino, em comparação aos oriundos de matrizes jovens.

Tabela 4. Peso do saco vitelino (SV), peso do fígado (FG), peso do esôfago + papo (EP), peso do próventrículo + moela (PM), peso dos intestinos delgado e grosso (PI), comprimento dos intestinos delgado e grosso (CI) e comprimento total do sistema digestivo (CSD) de pintos oriundos de ovos de matrizes com diferentes idades e submetidos ou não à inoculação de proteína isolada de soja

\begin{tabular}{|c|c|c|c|c|c|c|c|}
\hline \multirow{2}{*}{ Tratamentos } & \multicolumn{7}{|c|}{ Variáveis } \\
\hline & SV (g) & $\mathrm{FG}(\mathrm{g})$ & E P (g) & PM (g) & PI (g) & $\mathrm{CI}(\mathrm{cm})$ & $\mathrm{CSD}(\mathrm{cm})$ \\
\hline \multicolumn{8}{|l|}{ Soluções inoculadas } \\
\hline Ovo íntegro & 3,11 & 1,01 & 0,63 & 2,41 & 1,47 & 37,60 & 45,21 \\
\hline Placebo & 3,25 & 1,18 & 0,61 & 2,41 & 1,37 & 34,50 & 43,15 \\
\hline $1 \%$ P.I.S. & 4,31 & 1,07 & 0,63 & 2,11 & 1,42 & 36,90 & 46,25 \\
\hline 2\% P.I.S. & 4,73 & 1,18 & 0,67 & 2,39 & 1,23 & 38,30 & 47,55 \\
\hline \multicolumn{8}{|l|}{ Idade da matriz } \\
\hline Aves jovens & $3,15 b$ & 1,03 & 0,63 & 2,39 & $1,28 b$ & $34,95 b$ & $43,20 b$ \\
\hline Aves velhas & $4,55 \mathrm{a}$ & 1,08 & 0,64 & 2,25 & $1,46 \mathrm{a}$ & $38,70 \mathrm{a}$ & $47,96 \mathrm{a}$ \\
\hline \multicolumn{8}{|l|}{ Efeito } \\
\hline Soluções inoculadas & $0,08^{\text {ns }}$ & $0,17^{\mathrm{ns}}$ & $0,88^{\text {ns }}$ & $0,19^{\text {ns }}$ & $0,34^{\mathrm{ns}}$ & $0,11^{\mathrm{ns}}$ & $0,17^{\mathrm{ns}}$ \\
\hline Idade da matriz & $0,01 *$ & $0,44^{\mathrm{ns}}$ & $0,88^{\text {ns }}$ & $0,20^{\text {ns }}$ & $0,07 *$ & $0,01 *$ & $0,01 *$ \\
\hline Interação & $0,25^{\text {ns }}$ & $0,05^{\mathrm{ns}}$ & $0,35^{\mathrm{ns}}$ & $0,16^{\mathrm{ns}}$ & $0,38^{\text {ns }}$ & $0,32^{\text {ns }}$ & $0,25^{\text {ns }}$ \\
\hline $\mathrm{CV}(\%)$ & 4,26 & 21,90 & 26,55 & 15,43 & 21,73 & 9,73 & 9,82 \\
\hline
\end{tabular}

A inoculação de proteína isolada de soja afetou significativamente $(\mathrm{P}<0,05)$ o ganho de peso e o peso final, em que pintos oriundos de ovos inoculados apresentaram menores resultados. Estes corroboram o estudo de Leitão et al. (2010), que, ao avaliarem o desempenho de pintos oriundos de ovos inoculados ou não com carboidratos pelo período de um a sete dias de idade, observaram que a suplementação in ovo apresentou dados inferiores aos ovos íntegros. Em contrapartida, ambos os resultados diferem dos encontrados por Tako et al. (2004), que, ao suplementarem um complexo de carboidratos em ovos embrionados, constataram que pintos oriundos de ovos inoculados atingiram pesos superiores àqueles de ovos íntegros após três, sete e 10 dias de avaliação.

Todavia, vale ressaltar que estes autores utilizaram ovos com pesos superiores aos dos ovos utilizados neste estudo. Dessa forma, é possível que pintos provenientes de ovos mais pesados possam responder melhor à suplementação de nutrientes em ovos do que os pintos de baixo peso.

$\mathrm{Na}$ idade da matriz, verificou-se efeito significativo $(\mathrm{P}<0,05)$ sobre peso inicial, peso final, ganho de peso e conversão alimentar dos pintos (Tab. 5), em que pintos oriundos de matrizes velhas apresentaram melhor desempenho. Nesse contexto, Pinchasov e Noy (1993) afirmam que pintos derivados de matrizes jovens tendem a apresentar um desempenho inferior aos derivados de matrizes velhas, principalmente devido a menores quantidades de albúmen e gema em ovos de matrizes jovens, o que compromete o desenvolvimento embrionário pela menor disponibilidade de nutrientes. Maiorka (2002), ao trabalhar com matrizes de diferentes idades (30 e 60 semanas), afirma ainda que pintos de matrizes velhas possuem o trato 
gastrintestinal mais desenvolvido quando comparados aos de matrizes jovens, fato que contribui para um melhor desempenho e adaptação destes à alimentação exógena póseclosão.

Outrora, verificou-se efeito significativo $(\mathrm{P}<0,05)$ na interação entre os fatores sobre a conversão alimentar (Tab. 6), em que os pintos oriundos de matrizes jovens provenientes de ovos inoculados apresentaram melhores resultados. Nesse contexto, constata-se que a inoculação pode estimular precocemente $o$ desenvolvimento do TGI de pintos oriundos de matrizes jovens e, assim, auxiliar na regulação do consumo de ração, na melhora do aproveitamento dos nutrientes e, consequentemente, no ganho de peso. Vale ressaltar ainda que o consumo de ração, mesmo não tendo apresentado diferenças significativas $(\mathrm{P}>0,05)$, é parâmetro diretamente relacionado aos resultados de conversão alimentar e à resposta fisiológica objetivada no desempenho das aves.

Tabela 5. Médias de pesos inicial (PI), final (PF), ganho de peso (GP), consumo de ração (CR), conversão alimentar (CA) e viabilidade (Via) de pintos oriundos de ovos inoculados ou não com proteína isolada de soja

\begin{tabular}{|c|c|c|c|c|c|}
\hline & PI (g) & PF (g) & CR (g/ave/dia) & GP (g) & $\mathrm{CA}(\mathrm{g} / \mathrm{g})$ \\
\hline \multicolumn{6}{|l|}{ Soluções inoculadas } \\
\hline Ovo íntegro & 33,18 & $94,88 \mathrm{a}$ & 13,08 & $61,70 \mathrm{a}$ & 0,13 \\
\hline Placebo & 33,32 & $88,72 \mathrm{ab}$ & 13,46 & $55,40 \mathrm{ab}$ & 0,15 \\
\hline $1 \%$ P.I.S. & 32,72 & $84,80 \mathrm{~b}$ & 11,89 & $52,08 \mathrm{~b}$ & 0,14 \\
\hline 2\% P.I.S. & 32,52 & $85,99 \mathrm{~b}$ & 12,55 & $53,47 \mathrm{~b}$ & 0,14 \\
\hline \multicolumn{6}{|l|}{ Idade da matriz } \\
\hline Aves jovens & $31,20^{\mathrm{b}}$ & $84,75 b$ & 11,86 & $61,70 \mathrm{a}$ & $0,14 \mathrm{a}$ \\
\hline Aves velhas & $34,67^{\mathrm{a}}$ & $92,32 \mathrm{a}$ & 13,63 & $55,40 \mathrm{ab}$ & $0,16 b$ \\
\hline \multicolumn{6}{|l|}{ Efeito } \\
\hline Soluções inoculadas & $0,42^{\mathrm{ns}}$ & $0,01 *$ & $0,88^{\mathrm{ns}}$ & $0,01 *$ & $0,12^{\mathrm{ns}}$ \\
\hline Idade da matriz & $0,01 *$ & $0,01 *$ & $0,23^{\mathrm{ns}}$ & $0,05^{*}$ & $0,05^{*}$ \\
\hline Interação & $0,28^{\mathrm{ns}}$ & $0,25^{\mathrm{ns}}$ & $0,26^{\mathrm{ns}}$ & $0,39^{\text {ns }}$ & $0,01 *$ \\
\hline $\mathrm{CV}(\%)$ & 3,69 & 6,97 & 3,67 & 11,38 & 9,71 \\
\hline
\end{tabular}

Tabela 6. Interação sobre a conversão alimentar de pintos oriundos de ovos de matrizes com diferentes idades inoculados ou não com proteína isolada de soja

\begin{tabular}{lcc}
\hline \multirow{2}{*}{ Soluções inoculadas } & \multicolumn{2}{c}{ Idade da matriz } \\
\cline { 2 - 3 } & \multicolumn{2}{c}{ Conversão alimentar $(\mathrm{g} / \mathrm{g})$} \\
\hline Ovo íntegro & $0,14 \mathrm{Aab}$ & $0,14 \mathrm{Aa}$ \\
Placebo & $0,16 \mathrm{Bb}$ & $0,15 \mathrm{ABab}$ \\
$1 \%$ P.I.S. & $0,13 \mathrm{Aa}$ & $0,16 \mathrm{Bb}$ \\
2\% P.I.S. & $0,13 \mathrm{Aa}$ & $0,16 \mathrm{Bb}$ \\
\hline \multicolumn{1}{c}{ CV $(\%)$} & & \\
\hline
\end{tabular}

CV - Coeficiente de variação. * Médias seguidas por letras diferentes, maiúsculas na linha (efeito entre idades) e minúsculas na coluna (efeito entre soluções) diferem entre si pelo teste de Tukey a 5\%.

\section{CONCLUSÕES}

Conclui-se, portanto, que a inoculação in ovo de proteína isolada de soja apresentou efeito positivo sobre a relação pinto-ovo, porém sem proporcionar melhora nos rendimentos de incubação, no desenvolvimento dos órgãos e no desempenho de pintos na fase pré-inicial. A idade da matriz influenciou diretamente as variáveis avaliadas, em que ovos oriundos de aves velhas apresentaram maior peso, maior mortalidade embrionária na fase tardia, pintos mais pesados e pior conversão alimentar. 


\section{REFERÊNCIAS}

ALVES, L.A. Recursos ergogênicos nutricionais. Rev. Mineira Educ. Fís., v.10, p.23-50, 2002.

CAMPOS, A.M.A.; GOMES, P.C.; ROSTAGNO, H.S. Nutrição in ovo de frangos de corte. Rev. Eletrôn. Nutr., v.7, p.1304-1313, 2010.

CAMPOS, A.M.A.; ROSTAGNO, H.S.; GOMES, P.C. et al. Efeito da inoculação de soluções nutritivas in ovo sobre a eclodibilidade e o desempenho de frangos de corte. Rev. Bras. Zootec., v.40, p.17121717,2011

FREITAS, L.W.; GARCIA, R.G.; ALMEIDA PAZ, I.C.L et al. Nutrição in ovo - inovação no processo de incubação. VI SIMPÓSIO DE CIÊNCIAS DA UNIVERSIDADE ESTADUAL PAULISTA, 6. 2010. Dracena, Anais.... Dracena, São Paulo, UNESP, 2010. 3p.

IPEK, A.; SAHAN, U.; YILMAZ, B. The effects of in ovo ascorbic acid and glucose injection in broiler breeder eggs on hatchability and chick weight. Arch. Geflugelk., v.68, p.132-135, 2004.

JOCHEMSEN, P.; JEURISSEN, S.H.M. The location and uptake of in ovo injected soluble and particulate substances in the chicken. Poult. Sci., v.81, p.18111817, 2002.

LEITÃO, R.A.; LEANDRO, N.S.M.; STRINGHINI, J.H. et al. Inoculação de maltose, sacarose ou glicose em ovos embrionados de baixo peso. Acta Sci. Anim. Sci., v.32, p.93-100, 2010.

LUQUETTI, B.C.; GONZALES, E.; BRUNO, L.D.G. et al. Egg traits and physiological neonatal chick parameters from broiler breeder at different ages. Rev. Bras. Ciênc. Avíc., v.6, p.13-17, 2004.

MAIORKA, A. Efeito da idade da matriz e do agente trófico (glutamina) sobre o desenvolvimento da mucosa intestinal e atividade enzimática do pâncreas de pintos de corte na primeira semana. 2002. 103f. Tese (Doutorado em Zootecnia) - Universidade Estadual Paulista, Jaboticabal, SP.

MAIORKA, A.; SANTIN, E.; FISCHER, A.V. et al. Desenvolvimento do trato gastrointestinal de embriões oriundos de matrizes pesadas de 30 e 60 semanas de idade. Rev. Bras. Ciênc. Avíc., v.2, p.141-148, 2000.

McDANIEL, G.R.; ROLAND, D.A.; COLEMAN, M.A. The effect of egg shell quality on hatchability and embryonic mortality. Poult. Sci., v.58, p.10-13, 1979.
McLOUGHLIN, L.; GOUS, R.M. Efecto del tamaño del huevo en el crecimiento pre y post natal de pollitos de engorde. Avicul. Prof., v.18, p.24-29, 2000.

MEIJERHOF, R.; NOORDHUIZEN, J.P.T.M.; LEENSTRA, F.R. Influence of Preincubation Treatment on Hatching Results of Broiler Breeder Eggs Produced at 37 and 59 weeks of Age. Br. Poult. Sci., v.35, p.249-257, 1994.

OHTA, Y.; KIDD, M.T. Optimum site for in ovo amino acids injection in broiler breeders eggs. Poult. Sci., v.80, p.1425-1429, 2001.

PEDROSO, A.A.; CHAVES, L.S.; LOPES, K.L.A.M. et al. Inoculação de nutrientes em ovos de matrizes pesadas. Rev. Bras. Ciênc. Avíc., v.35, p.2018-2026, 2006.

PINCHASOV, Y.; NOY, Y. Comparison of post-hatch holding time and subsequente early performance of broiler chicks and turkey poults. Br. Poult. Sci., v.34, p.110-120, 1993.

ROSTAGNO, H.S.; ALBINO, L.F.T.; DONZELE, J.L; GOMES, P.C. Tabelas brasileiras para aves $e$ suínos: composição de alimentos e exigências nutricionais. 2.ed. Viçosa: UFV, 2011. 186p.

SANTOS, T.T. Influência da inoculação $e$ ingredientes intra-ovo em aspectos produtivos $e$ morfológicos de frangos de corte oriundos de distintos pesos de ovos. 2007. 63p. Dissertação (Mestrado em Qualidade e Produtividade Animal) - Faculdade de Zootecnia e Engenharia de Alimentos da Universidade de São Paulo, Pirassununga, SP.

STATISTICAL analysis system. Version 9.2. Cary: SAS Institute 2008.

TAKO, E.; FERKET, P.R.; UNI, Z. Effects of in ovo feeding of carbohydrates and $\beta$-hidroxy- $\beta$ methylbutyrate on the development of chichen intestine. Poult. Sci., v.83, p.2023-2028, 2004.

UNI, Z.; FERKET, P.R. Methods for early nutrition and their potential. World's Poult. Sci. J., v.60, p.101$111,2004$.

VICK, S.V.; BRAKE, J.; WALSH, T.J. et al. Relationship of incubator humidity and flock age to hatchability of broiler hatching eggs. Poult. Sci., v.72, p.251-258, 1993.

VIEIRA, S.L., MORAN JR., E.T. Broiler chicks hatched from egg weight extremes and diverse breeder strains. J. Appl. Poult. Res., v.7, p.392-402, 1998. 